\title{
DRAWING ALICANTE / DRAWING PALERMO. AN EXPERIENCE OF TEACHING INNOVATION WITHIN THE ERASMUS+ PROGRAM
}

\author{
P.J. Juan Gutiérrez ${ }^{1}$, V. Garofalo \\ ${ }^{1}$ University of Alicante (SPAIN), \\ ${ }^{2}$ University of Palermo (ITALY)
}

\begin{abstract}
The present research work exposes the results of a teaching innovation experience carried out in connection with a mobility between Spain and Italy. We strongly believe that an inter-university experience, within the framework of a traditional academic program, can help students to learn new ways of understanding teaching, drawing, the profession and the architecture itself. The starting hypothesis defends the self-assessment as a method to build a critical positioning with respect to the drawing.

To understand the research, it is necessary to explain the process: The students of the University of Alicante, within the framework of the Master of Architecture, have had the opportunity to propose a series of exercises to students of the first course of Architecture and Urbanism of the University of Palermo. The objective has been multiple. On one hand, the ability of students from Alicante to build statements that, in a graphic context, were coherent and could adapt to a specific academic year. On the other hand, the solvency of the students of Palermo to solve small graphical exercises outside their current educational program. But the experience does not end at this point. Once the exercises have been done, the students have played the role of teacher to assess, with the pertinent justification, the results of their proposals, and have sent their considerations and advice to their foreign colleagues.
\end{abstract}

The conclusions of the work, as will be argued during the detailed analysis of the results, are as encouraging as they are exciting. The possible union links that were intuited at the beginning of the work have ended up constituting firm bridges of communication. Thanks to these bridges the students, exercising a role with more responsibility than the simple learner, have been able to maximize their abilities to help, in a graphic context, to build a shared criterion that, surely, will lay the foundations of their maturity as draftsmen and, therefore, as architects.

Keywords: Graphic expression, mobility, Erasmus+.

\section{INTRODUCTION}

The present research work exposes the results of a teaching innovation experience carried out in connection with a mobility between Spain and Italy. We strongly believe that an inter-university experience, within the framework of a traditional academic program, can help students to learn new ways of understanding teaching, drawing, the profession and the architecture itself. The starting hypothesis defends the self-assessment as a method to build a critical positioning with respect to the drawing.

To understand the research it is necessary to explain the process: The students of the University of Alicante, within the framework of the Master of Architecture, have had the opportunity to propose a series of exercises to students of the first course of Architecture and Urbanism of the University of Palermo. The objective has been multiple. On one hand, the ability of students from Alicante to build statements that, in a graphic context, were coherent and could adapt to a specific academic year. On the other hand, the solvency of the students of Palermo to solve small graphical exercises outside their current educational program. But the experience does not end at this point. Once the exercises have been done, the students have played the role of teacher to assess, with the pertinent justification, the results of their proposals, and have sent their considerations and advice to their foreign colleagues.

The conclusions of the work, as will be argued during the detailed analysis of the results, are as encouraging as they are exciting. The possible union links that were intuited at the beginning of the work have ended up constituting firm bridges of communication. Thanks to these bridges the students, exercising a role with more responsibility than the simple learner, have been able to maximize their abilities to help, in a graphic context, to build a shared criterion that, surely, will lay the foundations of their maturity as draftsmen and, therefore, as architects. 


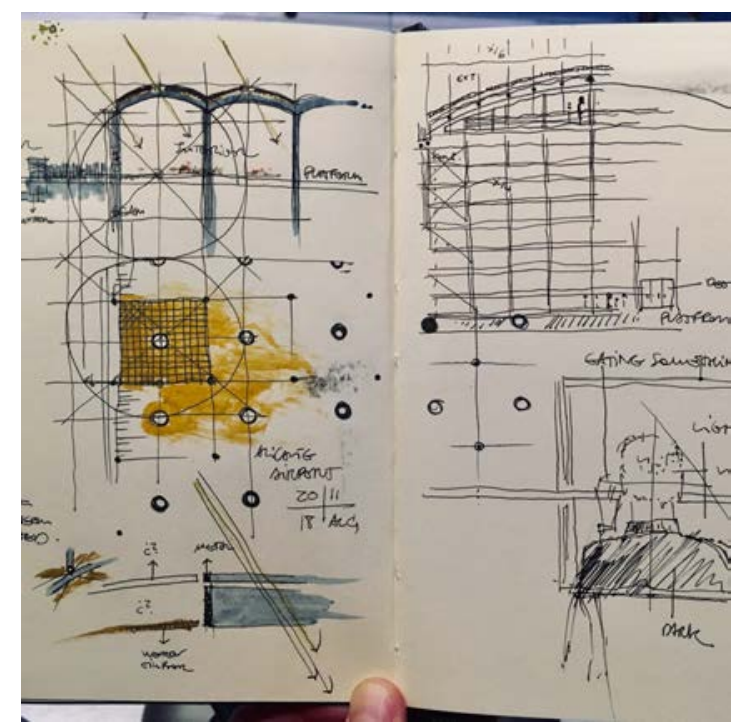

Figure 1. Drawing of the trip Alicante-Palermo. The teacher as a spectator of experience I.

\section{METHODOLOGY}

To clarify both the scope of the proposal and the development process carried out we will guide the work in three sections, namely: Alicante, Palermo and Connections.

\subsection{Alicante}

The context in which the teaching experience in Spain is carried out is the following: these are graduates in Fundamentals of Architecture who finish the cycle with studies in the Master of Architecture, which allow to practice the profession in Spain. It will be them, novice architects at the same time as experienced students, to whom the first challenge is proposed in the form of an exercise: to build an enunciation aimed at Italian first-year students. The experience is intended to be enriching on several levels.

In the first place, the student will have the opportunity to replace his/her teaching role with a role, although temporary and symbolic, of a teacher. Changing the point of view from which to think the graphic exercises will generate an empathy that, once finished the route through the itinerary of the graphic of the university studies, will begin to conform the criterion of evaluation of the visual that will accompany the student during his/her career path. Thinking about an exercise as a tool to achieve a goal different from its solution is a way to continue developing graphic capabilities beyond the preestablished paths. The importance of the spatial interest and construction of the architectural forms that integrate the proposal (Bravo and Gutiérrez 2011) must be complemented with an objective criterion of the graphic (Juan and Oliva 2017) that, previously defined, helps to establish a reference and a context of application and consensual validity.

Secondly, there is implicit a temporal reflection. Self-criticism. Reflective. The fourth-year Architecture student will think about the intellectual condition of the first-year student and, collaterally or unconsciously (it makes no odds) in his/her own condition as a student in the past. Considering its limitations, its achievements and, in short, the conjuncture that constitutes the personal context of each one, it must think about an exercise that will inevitably be positioned critically in relation to those that it had to confront itself previously.

The third level of conscious reflection that arises with the experiment has to do, of course, with physical distance and, therefore, cultural difference. The exercise may consider the spatial context as a characteristic of itself. Either to think about the space itself (Palermo), the exterior (Alicante) or, simply, to exercise a series of graphic competences independent of the location (but always dependent on culture, as we will see), students must take into account this third concept in his proposal: the spatial one. 


\subsection{Palermo}

At the University of Palermo we find a teaching context in which the student is in his/her first year of university studies oriented to urban planning, on the one hand, or architecture, on the other. From his point of view, the exercise itself does not imply anything unprecedented or new since, in some way, it is presented as part of the tools and challenges that are supposed to be the current studies. The teacher presents them as their own and, unless the specific statement of the exercise is related in some way to the location from which they are conceived (Alicante), could be easily confused with the previous or the following. Where is the novelty at this point? In the content: being the fruit of a reflection made at the end of a regulated itinerary of learning the graphic, the student from Alicante who is in the role of the teacher will inevitably synthesize what has been learned to reconfigure it (in part) as a question. In the same way that it is impossible to establish the observation of the experiment without intervening in it, the question itself (in this case enunciated or statement) contains implicit the possible answers. Moreover, a good teacher must be aware of the territories in which the response (or answers) required will be developed. The importance of the experiment for the University of Palermo lies in the internationalization of the teaching proposal: the students of Palermo have an opportunity for growth because they can learn another didactic approach, thanks to the teacher of the University of Alicante, and the application of this approach thanks to the exercises proposed by the students of the same university. The innovation, seen from the Italian university, is in the foreign character of the statement and, therefore, in the territory of possibility of its results more than in the type of drawing with which, in relation for example to the Manual of architectural drawing (Ching 1986), position the question.

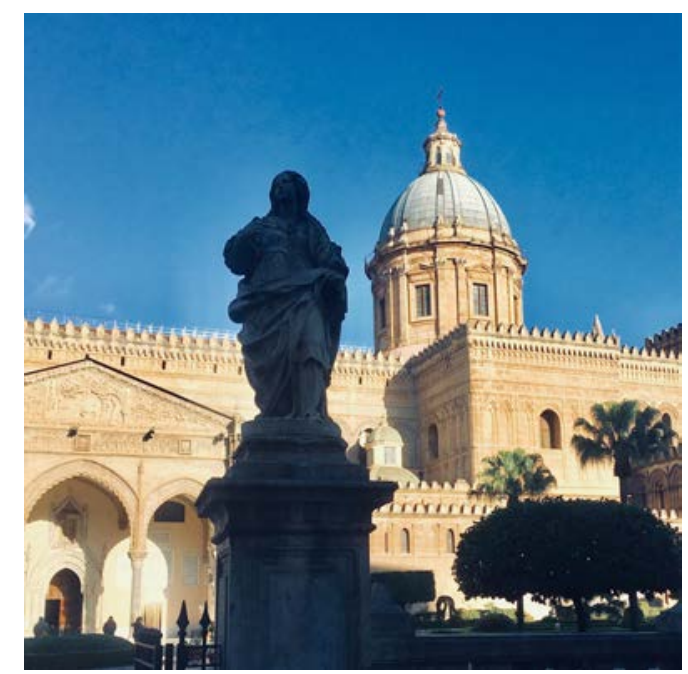

Figure 2. Photograph of the trip Alicante-Palermo.

The teacher as an observer of the experience II.

\subsection{Connexions}

Each one of the sections considered in the previous epigraphs will have its own repercussion during the consideration established by the links and the proposed repercussions. The union, the link, is fundamentally double. On the one hand we have the one that connects Alicante with Palermo, institutionally and personally but, on the other, we have the one that does the same between the fourth course of architecture and the first. Nevertheless, and being coherent with the previous discourse, we will differentiate between the following changes: Role, Time, Space, Context.

\subsubsection{Change of Role}

As we have explained above, the experienced students of Alicante will develop their own work as teachers. They will not only think about the statements themselves, but they will have to justify a criterion of correction and objective evaluation of the results. In this way, the Italian students will have the certainty that the proposal has the necessary guarantees so that the learning after their experiment is assured. But from the point of view of connections, the problem does not end here. As in a set of transparencies, each group of Italian students is assigned a foreign teacher who is the projection of a student from Alicante. This fact, known to them, generates, we believe, an empathic climate in which the student performs an exercise with the conditions imposed by someone who, like him, is immersed in a university learning process and, therefore, a teacher, and here is the key, it could be himself. As Fraile Aranda defends this self-evaluation 
among students develops the critical spirit, responsibility and autonomy, as well as stimulate meaningful learning. (Fraile 2010: 16)

\subsubsection{Change of time}

The aforementioned reflection that the Alicante student performs is part of a broader, complex and interesting process: identification. The distance is, needless to say, mutual. Four years ago the student from Alicante was in the situation of Italian ones. Within four years the palermitano student will be in the same situation as the Spanish one. They are part of the same group. They are, in some way, the same element. They have the same tools. The mirror by means of which, with transparencies, they guess the student after the teacher, he returns now, in addition, his/her own image.

\subsubsection{Change of space}

The result, in Alicante from Palermo or in Palermo from Alicante, will always be conditioned by the distance that exists between both cities. Regardless of where the graph is located, the drawing occurs in a different place from that of its evaluation. To the spatial vision that is supposed to any process of graphic representation will be added the spatial consideration derived from its international condition.

\subsubsection{Change of Context}

To the previous modifications, namely, of identity, temporal and spatial, the contextual one is added, since the experience, in addition to intercontinental, is intercultural. This fact will be evident not only at the beginning of the process (during the establishment of the statement) but also at the end of the process, that is, during the evaluation of the results. Although the scope of language and the tool that graphic expression means go beyond the limits that define languages, the impact that culture exerts on drawing is deeper than the result offered by the visual. The latent conceptual tensions that result from the detailed concrete conception of the exercise face the lines of thought after the resolution of the same. As if it were an iceberg, the visual result means a small part of the process, since the theoretical concepts that produce and that cement each decision or, what is the same, each line, have their roots in each one of the intervening agents and, therefore, in their culture.

\section{RESULTS}

Based on a general approach, we can classify the results based on three different positions: Statement, solution and experience.

\subsection{Statement}

The statements are classified into two large blocks, namely Spanish and Italian. These suppose, in themselves, one of the achievements of the experience that concerns us. Not only do they reorder reality according to the student's approach, but they also propose new techniques to deal with representation always from a practical point of view. Following the thought of John Berger the contemporaneity allows us to reorganize the role of images (Berger 1972) ... also those of the statements.

\subsubsection{Spanish statements}

Some students, exercising the role of teachers when structuring a statement, have decided to place their exercise in the scope of their own final Master's work. In this way, the statements entitled "2 STAZIONE DI SERVIZIO", "3 CAMPO E CITTÀ" and "4 FLUSSI" propose to be resolved taking into account specific landscapes or places within the Spanish borders.
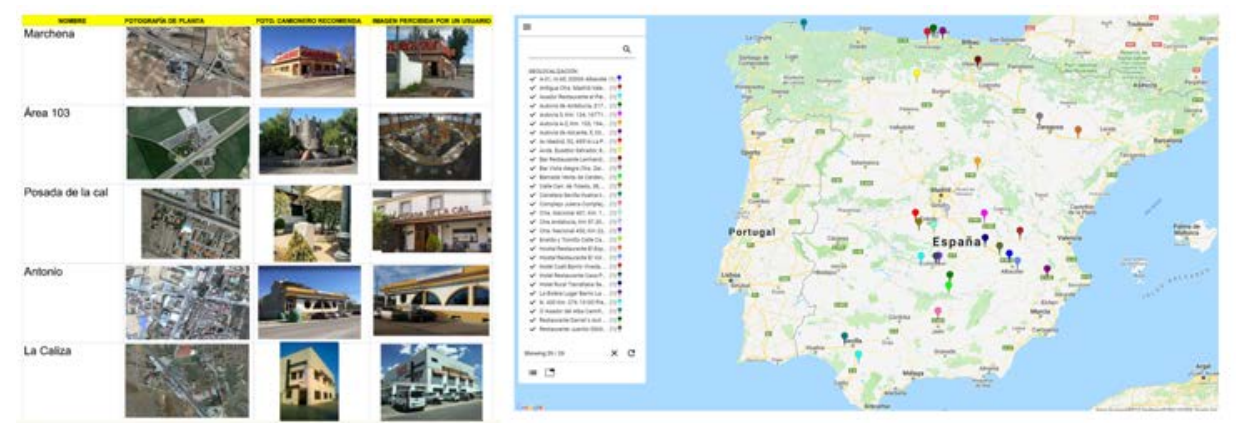

Figure 3. Fragment of statement "Stazione di servizio". 


\subsubsection{Italian statements}

The statements that, conceived from Alicante, propose to be solved and drawn in Italian territory are "1 PALERMO PUBBLICO PRIVATO", "5 FLAGS" and "6 PALERMO REDESIGNED". The students from Alicante here perform a conceptual extrapolation of the most important concerns of their own work to offer a series of exercises that, although delocalized, are ideologically similar to the problems they face in their own work. It is important to point out that some students knew how to take advantage of the opportunity to test a series of methodologies and experiences that, later, served as reference for their own processes.

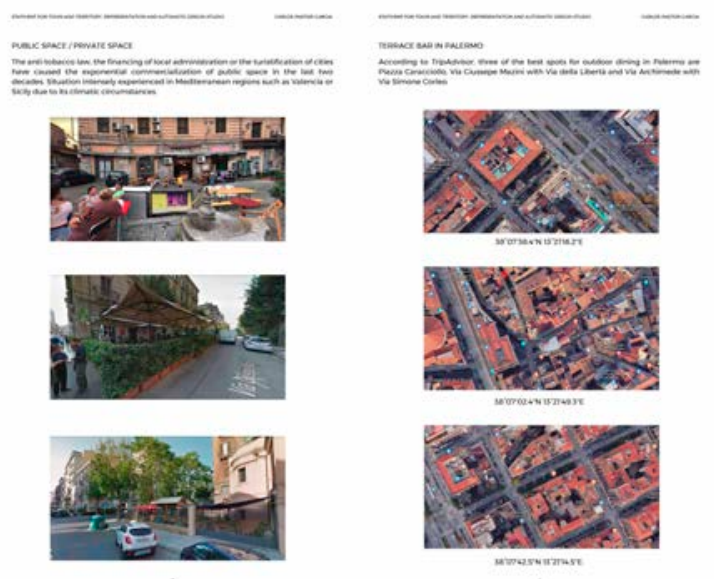

Figure 4. Fragment of statement "Palermo Pubblico Privato".

\subsection{Solution}

The solution of the exercises has served several purposes. In the first place, no doubt, the students have had the opportunity to face an external problem from a personal point of view. In the traditional way, the exercise has served as a tool to establish and agree on the student's knowledge. In the second place and as vehicles of communication that they are, they have determined (in a more or less eloquent way) a way of understanding the teaching of the graphic and, by extension, the profession and the architecture. Learning goes beyond resolution. The very configuration of the question, its focus and scope, determines and teaches new ways of understanding the city, architecture and drawing. It is difficult to overestimate the importance of this fact: the statements are, in themselves, manifestos and theoretical positions. In the third place, the creators of the enunciation have found a methodology to face everyday problems dependent on culture. That is to say, the solution of the exercises implies, before facing their evaluation and / or correction, a graphic and practical lesson although, as Alberto Giacometti pointed out "That's the terrible thing: the more one works on a picture, the more impossible it becomes to finish it "(Lord 1965: 11)

The examples have been numerous and, by the very extension of this research work, it is impossible to stop at all of them. We can, however, analyze a specific case that will serve as a model of what we intend to transmit with the investigation. One of the exercises, entitled "Palermo Pubblico Privato" and developed by the student Carlos Pastor García proposed, considering as a model the 1748 Map of Rome by Giambattista Nolli, to graph the part of the city of Palermo that was considered (each group of Italian students was autonomous to choose their own location according to their own considerations) and establish the following parallelism: when Nolli's map is working with the public and discovered space of the city including the ground floor of the most representative sacral spaces, the statement invited the student to create a drawing of the exterior public spaces (communication routes and streets) of the city of Palermo including the ground floor of the most representative recreational spaces. Specifically, those that are related, in one way or another, to the phenomenon known as "tardeo" and which is a playful trend through which spanish youth occupy public space and cafeterias between 4:00 p.m. and 8:00 p.m.

The result of the experiment ended up being a series of layers (figure 5) that, as filters and at an approximate scale of $1 / 500$, analyzed a facet of the city that, presumably, the students know better than anyone (because they are themselves the users of the spaces represented). Thus, the identification by colors of the public spaces discovered follows a rigorous drawing of the ground floor of the restaurants, cafeterias and bars in the area. To finish the exercise the students had drawn a series of legends in 
which they indicated semaphores and crosswalk, bins and bollards, ... etc. In short, the intended drawing of the ground floors of the playful spaces of Palermo, was transformed into an exhaustive analysis of the parameters that, in addition to the architecture, shapes the identity of a neighborhood. As one who gives hare for a cat (the Spanish expression "cat for hare" is used to denounce a result below the expectation) Italian students, theoretically less prepared than spanish ones because of their age, were able to represent graphically, with the tools of their level, the cultural identity that characterizes a place and that, otherwise, all the time goes unnoticed for foreigners and, of course, for tourists.

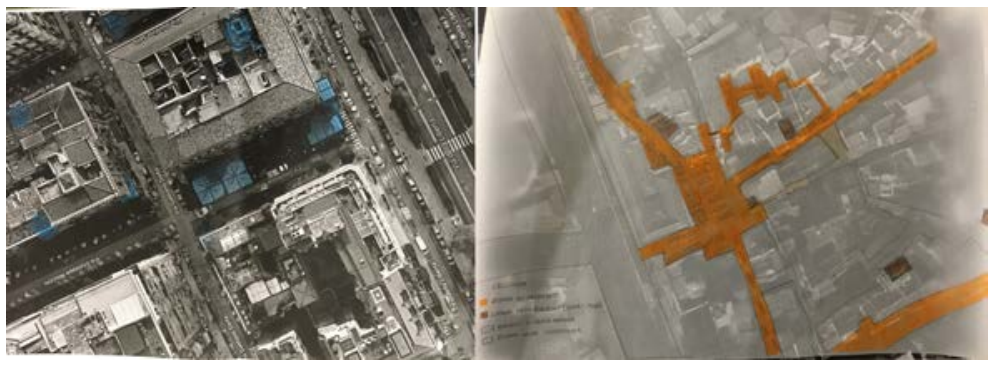

Figure 5. Fragment of part of the solution of "Palermo Pubblico Privato".

\subsection{Experience}

The evaluation of the experience has been made taking into account the impact that the project-based learning process has had. It must pass from an assessment of learning to an assessment for learning (Martínez et alters 2012: 336). More than teaching-learning research has shown a new way of acquiring knowledge that, without replacing the chosen method and in a timely manner, can help to build the necessary criteria that every good draftsman (and by extension architect) should exercise. Thus, this transversal process of learning-learning has served to guide a way of developing knowledge among students in an autonomous way: In Alicante the students have thought of some exercises to help, ultimately, help the first-year Italian students, with the simple fact of its resolution, concrete, specific and localized (that is, cultural), it has ended up reverberating and giving feedback to students from Alicante who, although more experienced in the itinerary of the graphic, have shown to possess a great interest in learning.

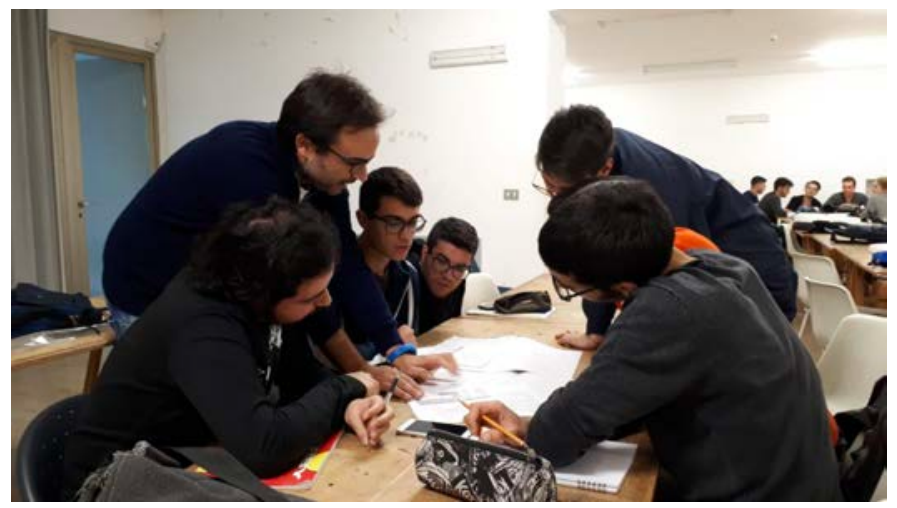

Figure 6. Vincenza Garofalo's photograph of a moment of the work process.

The main lines of learning that students of the Master of Architecture of Alicante have extracted at the end of the process can be summarized in three, namely: 1 . The importance of layers as a tool for organizing information, 2. The legend as an integral part of the drawing, 3. The precision of the drawing, its pertinence and its potentiality, is closely linked to the correct definition of the objectives that invoke it. Thus, and taking into account these three particular conclusions, the Alicante students restructured their own final works. In figure 7 we see a clear example of how learning can also arise from a different environment than the master class or the transfer of knowledge.

The main lines of learning for the students of Palermo are: 1 . The importance of being able to analyse and describe a territory even it is unknown through the tools of drawing, 2 . The awareness that the drawn information must be clear and understandable even by distant users, 3 The importance of careful and conscious observation for the correct representation and communication of the reading and analysis of urban spaces. 
Just a critical look at the world around us to extract recognizable and useful lessons. Using different layers to organize information consciously, extending the features of the drawing to the legend of the same and, finally, defining precisely the objectives to be met, are three unprecedented and unexpected conclusions that, as a result of the students themselves, have ended up define the nature of the subject "Graphic Tools for Urban Planning" this academic year and, surely, have solidly cemented their graphic criteria.

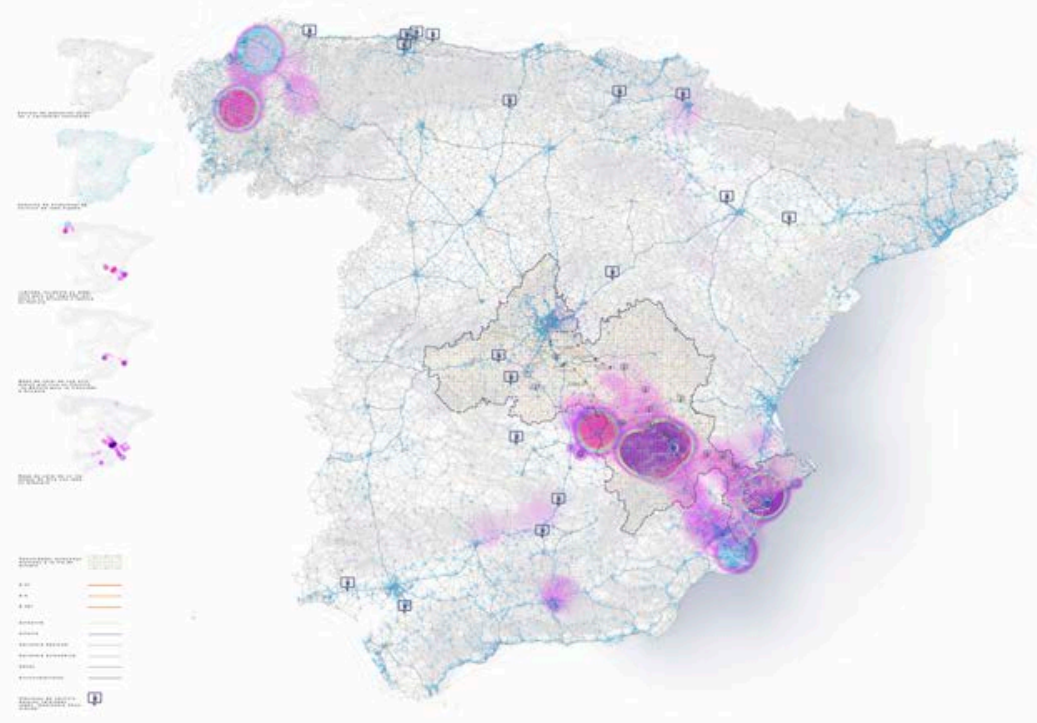

Figure 7. Result of experience Students María Jesús Salcedo and Belén Jaquero.

\section{CONCLUSIONS}

The main conclusions of the work are:

- The teaching-learning process can be carried out with excellence only, through the active and critical participation of students.

- The most important role of all that the teacher performs has to do with the synchronization and coordination of the patterns.

- The answers to the graphic problems are, first of all, cultural facts.

- The Erasmus + experience is evidenced as a tool that exceeds, by far, the initial considerations and pretensions.

- Every teacher is, simultaneously and in the same area of knowledge, a student. Every student is, asynchronically and in a different area of knowledge, a teacher.

\section{REFERENCES}

[1] Berger, John. 1972. "Modos de ver". Barcelona: Gustavo Gili

[2] Bravo De Laguna Socorro, A.; Gutiérrez Labory, E. (2011). Autogestión de la evaluación del dibujo del estudiante de arquitectura. A 'Univest 11'. Girona: Universitat.

[3] Ching, Frank (1986) "Manual del dibujo arquitectónico". Gustavo Gili. México

[4] Fraile Aranda, F. (2010), "La autoevaluación: una estrategia docente para el cambio de valores educativos en el aula", Ser Corporal, núm. 3.

[5] Juan Gutiérrez, P.; Oliva Meyer, J. "Criterios objetivos para la evaluación de lo gráfico" en RoigVila, Rosabel (coord.). Redes colaborativas en torno a la docencia universitaria. Alicante: Universidad de Alicante, Instituto de Ciencias de la Educación (ICE), 2017. ISBN 978-84-617-89733, pp. 422-432.

[6] Lord, J., 1965, Portrait of Giacometti, New York: the Museum of Modern Art. 
[7] Martínez Martínez, A., Cegarra Navarro, J. G., Rubio Sánchez, J. A., "Aprendizaje basado en competencias: una propuesta para la autoevaluación docente". Profesorado. Revista de Currículum y Formación de Profesorado [en linea] 2012, 16 (Mayo-Agosto). 